\title{
Spin-Polarized Electron Energy Loss Spectroscopy of High Energy, Large Wave Vector Spin Waves in Ultrathin fec Co Films on $\mathrm{Cu}(001)$
}

\author{
R. Vollmer, ${ }^{1, *}$ M. Etzkorn, ${ }^{1}$ P. S. Anil Kumar, ${ }^{1}$ H. Ibach, ${ }^{2}$ and J. Kirschner ${ }^{1}$ \\ ${ }^{1}$ Max-Planck-Institut für Mikrostrukturphysik, Weinberg 2, D-06120 Halle, Germany \\ ${ }^{2}$ Institut für Schichten und Grenzflächen ISG3, Forschungszentrum Jülich, D-52425 Jülich, Germany
}

(Received 6 June 2003; published 29 September 2003)

\begin{abstract}
The realm of high energy, large wave vector spin waves in ultrathin films and at surfaces is unexplored because a suitable method was not available up to now. We present experimental data for an $8 \mathrm{ML}$ thick $\mathrm{Co}$ film deposited on $\mathrm{Cu}(001)$ which show that spin-polarized electron energy loss spectroscopy can be used to measure spin-wave dispersion curves of ultrathin ferromagnetic films up to the surface Brillouin zone boundary.
\end{abstract}

DOI: 10.1103/PhysRevLett.91.147201

PACS numbers: 75.30.Ds, 75.50.Cc, 75.70.Ak

Spin waves are collective magnetic excitations of electron spins in magnetically ordered solids. While small wave vector spin waves are macroscopic in nature and can be described well by macroscopic, phenomenological theories, large wave vector spin waves require a microscopic description. The measurement of these large wave vector spin waves - until now a completely unexplored area for ultrathin films - gives direct access to microscopic quantities such as the exchange coupling constant and will allow a direct comparison to first principles calculations of dynamic properties of thin films. The dynamic and static properties of ultrathin films are often quantitatively and, in some cases, even qualitatively different from that of the bulk material. Therefore, experimental information on these large wave vector spin waves in ultrathin films is highly desired and may lead to fundamentally new insights into the spin dynamics in reduced dimensions in the future.

The properties of very small wave vector spin waves at surfaces and in thin films have been investigated extensively in the past by ferromagnetic resonance and Brillouin light scattering or optical time domain methods [1]. However, these methods are limited to wave vectors below $0.01 \AA^{-1}$. This problem is avoided in magnetic neutron scattering, where essentially spin waves within the whole Brillouin zone can be observed. For surfaces and ultrathin films, however, neutron scattering is not suitable since the interaction of neutrons with spin waves is so weak that the investigation of spin waves at surfaces or in a single thin film is practically out of reach [2].

Contrary to the collective spin-wave excitations, Stoner excitations can be described in a single particle picture: A Stoner excitation consists of an electron above the Fermi energy $E_{F}$ coupled to a hole below $E_{F}$ with opposite spin. They can be excited very efficiently by electron scattering, and their properties have been investigated extensively during the past 20 years by spinpolarized electron energy loss spectroscopy (SPEELS) [3-5]. Theory predicted a likewise observability of spin waves by SPEELS [6]. Later Gokhale et al. showed that the spin-wave cross section - though much smaller than that for phonons - is expected to be in a range where experimental detection is feasible [7]. However, until recently no evidence for spin waves was found in the experimental SPEEL spectra $[8,9]$. In this Letter we provide the first experimental proof that SPEELS indeed can be used to explore the high energy and high wave vector spin-wave region of thin films.

For the present experiments, a new high resolution electron energy loss spectrometer has been constructed [10]. A strained GaAs photocathode produces a (longitudinal) spin-polarized electron beam with a polarization parallel or antiparallel to the propagation direction of the photoelectron, depending on the helicity of the incident light [11]. The degree of spin polarization $P$ is $0.79 \pm 0.09$ in the present case. The photoemitted electrons pass a $90^{\circ}$ pre-monochromator and a $180^{\circ}$ monochromator with electrostatic deflection. Since the electrostatic deflection does not affect the spin orientation a transverse spinpolarized $e$-beam results. The scattering plane is chosen to be perpendicular to the spin orientation of the incident electrons and the total scattering angle is kept fixed at $90^{\circ}$ for most of the measurements. The electrons scattered from the surface are then energy analyzed without further determination of the spin direction. The total energy resolution in the experiments is about $40 \mathrm{meV}$ (FWHM). By rotating the crystal surface normal with respect to the incident angle $\theta$, the wave vector transfer parallel to the surface, $\Delta K=k_{f} \cos \theta-k_{i} \sin \theta$, can be changed. $k_{i}, k_{f}$ are the magnitude of the wave vectors of the incident and scattered electron, respectively (see inset of Fig. 1). Ultrathin face centered cubic (fcc) Co films have been prepared by molecular beam epitaxy of Co onto a $\mathrm{Cu}(001)$ single crystal at $300 \mathrm{~K}$. The thickness of the Co film has been calibrated by the oscillations with one monolayer period observed in the medium energy electron diffraction. For the data shown in this Letter the Co film was eight atomic layers thick. The Co film has been annealed at $450 \mathrm{~K}$ for $5 \mathrm{~min}$ to produce a smoother surface. It is known that for a thickness larger than 


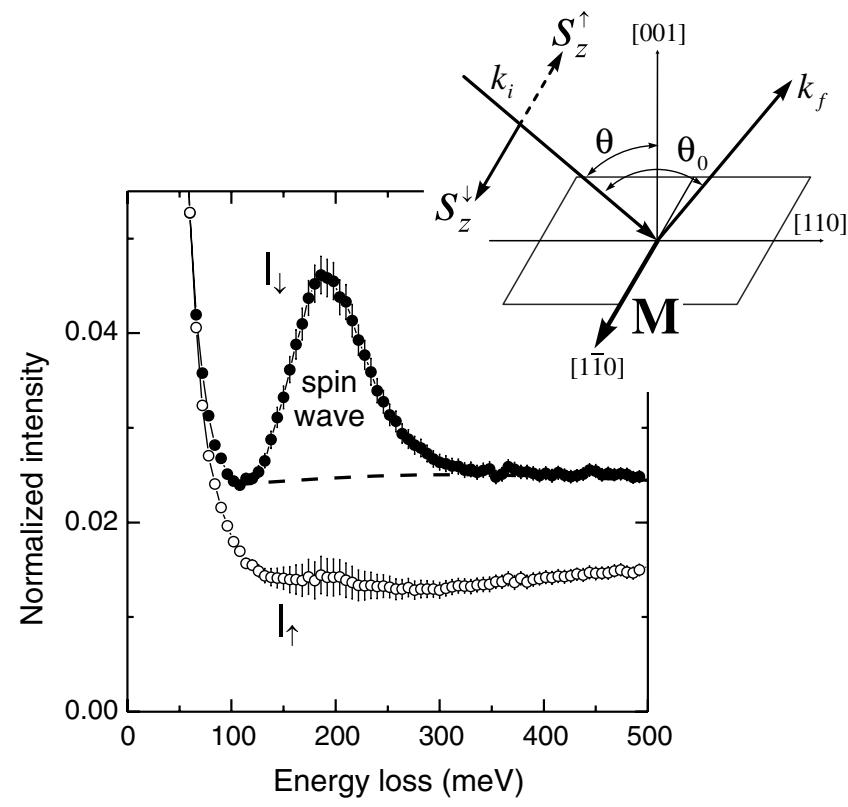

FIG. 1. SPEELS intensity spectra $I_{\uparrow}(\bigcirc)$ and $I_{\downarrow}(\bigcirc)$, normalized to the average intensity $\left(I_{\uparrow}+I_{\downarrow}\right) / 2$ at $0 \mathrm{meV}$ energy loss, for $E_{0}=6.7 \mathrm{eV}$ and $\Delta K=-0.87 \AA^{-1}$. The dashed line indicates qualitatively the contribution of Stoner excitations to $I_{\downarrow}$. The inset describes the scattering geometry: $S_{z}^{(\downarrow)}$ is the spin polarization of the incident beam, $M$ is the magnetization direction, $k_{i}, k_{f}$ are the wave vectors of the incident and scattered electron, $\theta$ is the incident angle, and $\theta_{0}=90^{\circ}$ is the angle between the monochromator and the detector.

4 ML these films are stable against pinhole formation and interdiffusion at $450 \mathrm{~K}$ [12]. After preparation, the Co

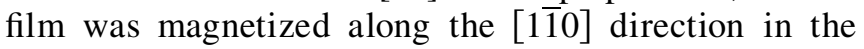
surface plane and perpendicular to the scattering plane. Thus, the polarization $S_{z}^{\downarrow(\uparrow)}$ of the incident electron beam was either parallel or antiparallel to the magnetization, respectively. Electron energy loss (EEL) intensity spectra for the polarization of the incident beam antiparallel $\left(I_{\uparrow}\right)$ and parallel $\left(I_{\downarrow}\right)$ to the magnetization were recorded. In the figures shown in this Letter, we have corrected the intensities for the incomplete polarization $P$ of the source.

The measurement of only the total scattered intensity without spin analysis of the scattered electrons does not allow one to determine whether the scattered electron has a spin orientation opposite to that of the incoming electron (spin-flip scattering) or it maintains its spin orientation (non-spin-flip scattering). However, as complete (low resolution) SPEELS experiments with spin analysis of the scattered electrons have shown, the difference in the scattering intensity $I_{\uparrow}$ and $I_{\downarrow}$ is caused mainly by a different scattering cross section for spin-flip scattering in the whole energy loss range [5,13]. The nonflip scattering cross section is only weakly dependent on the spin polarization of the incident electron. Therefore, a large difference in $I_{\uparrow}$ and $I_{\downarrow}$ is caused by strong spin-flip scattering.

For large energies of the incident electron, $E_{0}=25 \mathrm{eV}$, we observed merely a broad distribution with a maximum at about $0.8 \mathrm{eV}$ in the $I_{\downarrow}$ spectrum in agreement with
Ref. [13]. In that paper all the spin-flip scattering at $E_{0}=$ $25 \mathrm{eV}$ was attributed to Stoner pair creation in the sample. The situation changes dramatically for lower energies of the incident electrons. In Fig. $1 I_{\uparrow}$ and $I_{\downarrow}$ EEL spectra are shown for $E_{0}=6.7 \mathrm{eV}$ and $\Delta K=$ $-0.87 \AA^{-1}$. Out of the background of Stoner excitations a pronounced peak at about $195 \mathrm{meV}$ loss energy emerges in the $I_{\downarrow}$ spectrum, while no such peak is observable in the $I_{\uparrow}$ spectrum. We attribute the peak in the $I_{\downarrow}$ spectrum to the creation of a spin wave by the scattered electron: The spin wave reduces the magnetization of the sample and therefore changes its angular momentum. Therefore, the conservation of angular momentum requires, that only minority electrons, i.e., electrons which have their magnetic spin moment antiparallel to the magnetization, can excite the spin wave in the sample. This behavior distinguishes the spin-wave peak from nonmagnetic excitations such as vibration modes, for example.

As discussed above, we attribute most of the difference between $I_{\downarrow}$ and $I_{\uparrow}$ at large energy loss to the creation of Stoner excitations by incident minority spin electrons. Although not forbidden by a conservation law, for incident majority spin electrons the intensity of Stoner excitations is quite low because of the low density of available empty states in a strong ferromagnetlike Co. The dashed line in Fig. 1 extrapolates qualitatively the Stoner background toward lower energies. Obviously, Stoner excitations contribute significantly in the low energy loss range of spin waves as well. There is no apparent threshold of Stoner-pair excitation.

In Fig. 2, a series of EEL intensity spectra, in Fig. 2(a) $I_{\uparrow}$, and in Fig. 2(b) $I_{\downarrow}$, for various incident angles $\theta$, are shown for $E_{0}=6.7 \mathrm{eV}$ in the low energy loss range up to $400 \mathrm{meV}$. In Fig. 2(b) $\left(I_{\downarrow}\right)$ a well-defined peak emerges as a shoulder from the diffuse elastic peak and moves toward higher energy losses with increasing $|\Delta K|$. To emphasize the difference in the $I_{\uparrow}$ and $I_{\downarrow}$ spectra, usually an asymmetry $A=\left(I_{\downarrow}-I_{\uparrow}\right) /\left(I_{\downarrow}+I_{\uparrow}\right)$ is defined. It is plotted in Fig. 2(c) and shows the peak structure as well, most clearly at low $|\Delta K|$. The asymmetry at large energy losses is due to Stoner excitations. Their intensity increases toward larger wave vectors, as has been observed in the past $[5,13]$.

The dispersion curve of the spin waves is plotted in Fig. 3 as solid symbols. Because of the low energy $E_{0}$ of the incident electrons, the parallel wave vector transfer is limited to values below about $\pm 1.2 \AA^{-1}$ for an angle $\theta_{0}=$ $90^{\circ}$. Slightly higher wave vectors can be accessed by reducing $\theta_{0}$ to $80^{\circ}$, which allows measurement of spin waves in the next Brillouin zone (squares in Fig. 3). Despite the limited range of data points in the second Brillouin zone, it is clear that the dispersion curve is symmetric around the $\bar{X}$ point, as it is expected for a surface or thin film dispersion curve.

As a first attempt to describe the experimental data we have employed the most simple model, the Heisenberg 


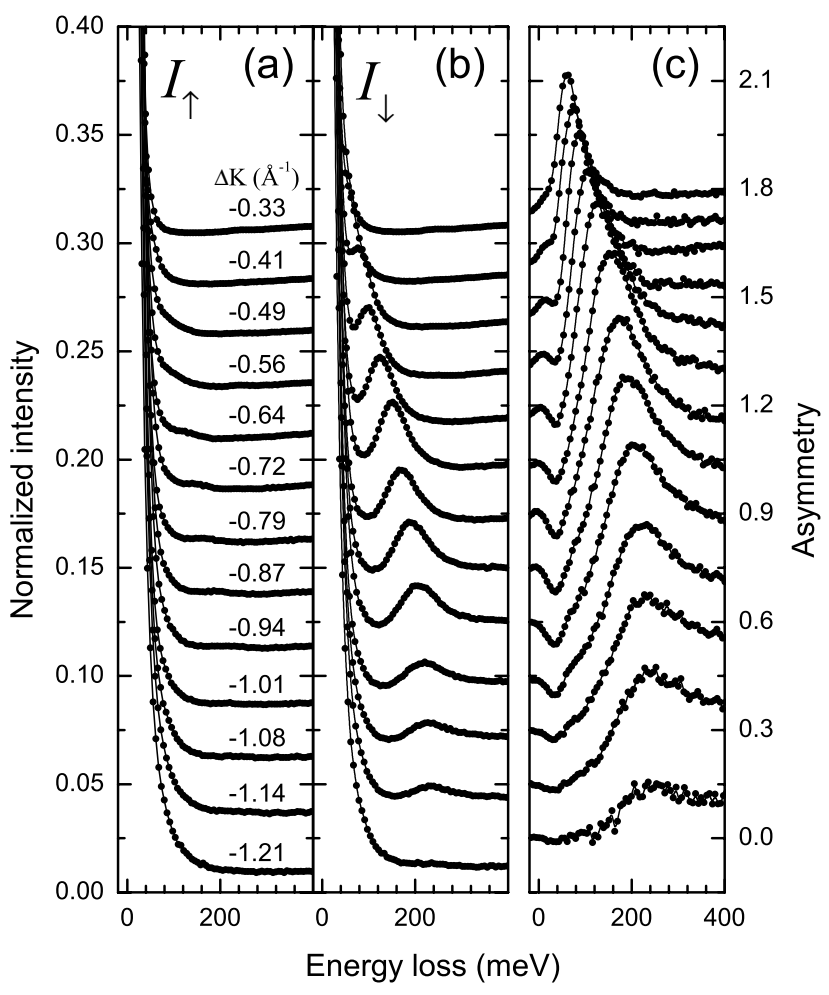

FIG. 2. Series of normalized SPEELS intensity spectra (a) $I_{\uparrow}$ and (b) $I_{\downarrow}$. (c) Asymmetry spectra for different angles of incidence, corresponding to the $\Delta K$ values plotted in panel (a). $E_{0}=6.7 \mathrm{eV}$. The intensity curves are offset by 0.025 and the asymmetry curves by 0.15 with respect to each other.

model with nearest neighbor (NN) interactions, $H=$ $-J \sum_{\langle i, j\rangle} S_{i} S_{j}$, to the fcc Co film with the exchange coupling constant $J$ as the only fit parameter. A surface mode exists in this model for a semi-infinite solid with a dispersion curve $\hbar \omega^{\text {(surf) }}(q)=8 J S\left(1-\cos \left(q a_{0}\right)\right)$ along the $\langle 110\rangle$ direction [15]. $q$ is the length of the wave vector of the spin wave, $a_{0}=2.55 \AA$ is the nearest neighbor distance, and $S$ is the magnitude of the spin per (primitive) unit cell. The fit of this curve to the measured SPEELS data yields $J S=15.0 \pm 0.1 \mathrm{meV}$. We estimate the total error including systematic errors to be less than $1 \mathrm{meV}$. The surface projected bulk states are plotted as the gray area in Fig. 3, as well using the same value of $J S$. From that it is clear that the surface mode at the surface zone boundary $(\overline{\mathrm{X}})$ is well below the bulk band edge. This is caused entirely by the reduced number of only eight nearest neighbors at the surface layer compared to 12 in the bulk. Unfortunately no experimental neutron data on fcc Co are available for these high energies to compare directly with. However, the value $J S=14.7 \pm 1.5 \mathrm{meV}$ derived from the available low energy neutron data on fcc Co (with $8 \% \mathrm{Fe}$, to stabilize the fcc phase at room temperature) match perfectly with the data derived from the SPEELS data [14]. Note that the bulk Brillouin zone along the $\langle 110\rangle$ direction ( $K$ point) is at a 1.5 times larger wave vector than that of the surface Brillouin zone $(\overline{\mathrm{X}})$ point.

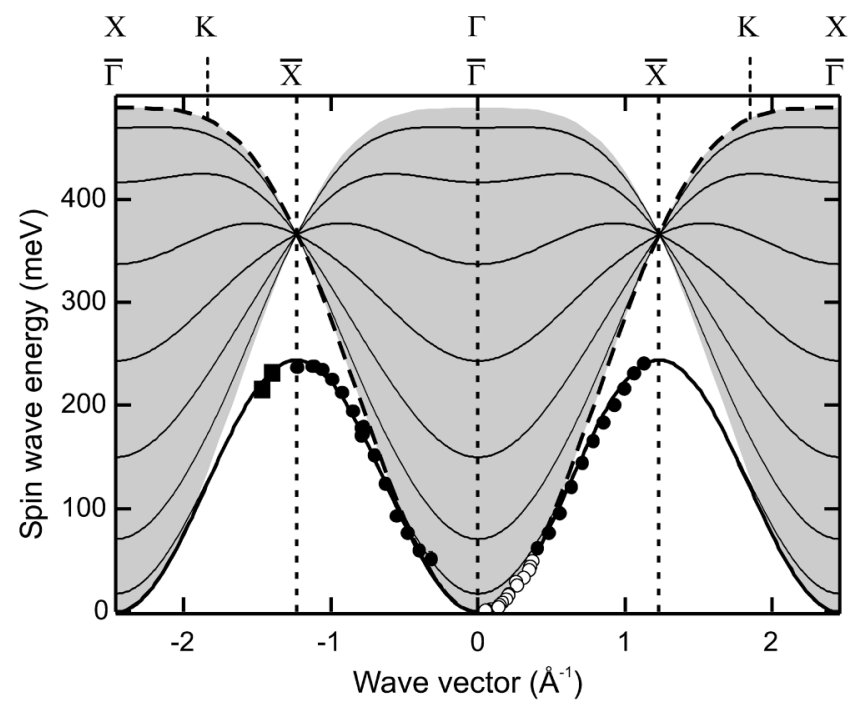

FIG. 3. Spin wave energy versus parallel wave vector transverse $\Delta K$ derived from the spin-wave peak position in the $I_{\downarrow}$ EEL spectra. $E_{0}=6.7 \mathrm{eV}, \theta_{0}=90^{\circ}(\mathbf{O}), \theta_{0}=80^{\circ}$ (ם). All lines and the gray area result from the NN Heisenberg model with $J S=15 \mathrm{meV}$ : The solid thick line is the surface mode of a semi-infinite solid and the gray area the surface projected bulk modes. The thin solid lines are the eight dispersion curves for an $8 \mathrm{ML}$ thick slab, from which the lowest mode nearly coincides with the surface mode. For comparison the available neutron data on fcc $\mathrm{Co}(8 \% \mathrm{Fe})$ along the [110] direction in the bulk are plotted as well $(\bigcirc$, Ref. [14]). The dashed line is the bulk spin-wave dispersion curve along the [110] direction using the same value for $J S$ as for the SPEELS data.

For an $8 \mathrm{ML}$ thin film, not only a single (surface) mode is expected but - within the Heisenberg model -8 spinwave branches. These are plotted as thin lines in Fig. 3. The lowest, "acoustic" branch nearly coincides with the surface mode. Therefore, an $8 \mathrm{ML}$ thick film is already a good approximation of a semi-infinite solid for the wave vector range above $0.3 \AA^{-1}$ as investigated here. In the spectra no obvious signs of the additional "optical" or "standing spin-wave" branches are visible. This points to the limitations of the Heisenberg model. The more sophisticated theory of Ref. [16] takes into account the interaction of the spin waves with Stoner excitations and finds fewer spin-wave branches than predicted by the Heisenberg model.

The spin-wave peaks in the spectra of Figs. 1 and 2 are significantly broadened. Corrected for the finite energy resolution of the instrument, a width ranging from about 40 to $75 \mathrm{meV}$ results. Attributing this width solely to the finite lifetime of the spin wave, this lifetime would be of the order of 10 fs only. However, it cannot be ruled out that the peak contains an unresolved optical mode of significant lower intensity, since the peak shape is slightly asymmetric. The "dressing up" of the spin wave with single particle excitations might be another possible reason for the increased width. 
The intensity of the spin-wave peak decreases by almost 2 orders of magnitude in the investigated $\Delta K$ range toward higher wave vectors. (Note, the spectra in Figs. 1 and 2 are normalized with respect to the diffuse elastic peak, which itself drops by about 1.5 orders of magnitude.) This decrease in the intensity and the broadening of the spin wave is concomitant with the increased intensity of the Stoner excitations which suggests that indeed the short lifetime of the spin wave at larger $\Delta K$ is due to the enhanced decay of the spin wave into Stoner excitations.

Available $a b$ initio calculations for fcc bulk Co predict spin-wave energies of $525 \mathrm{meV}$ [17] or $400 \mathrm{meV}$ [18] at the wave vector $q=1.23 \AA^{-1}(1 / \sqrt{2}, 1 / \sqrt{2}, 0)$ (corresponding to the $\bar{X}$ point of the surface Brillouin zone). In the NN Heisenberg model, the spin-wave energy for the bulk is 1.5 times that for the surface at $\bar{X}$. Assuming that this factor holds approximately for the more sophisticated calculations of Refs. $[17,18]$ the deviation of these theories to our result is $30 \%$ and $8 \%$, respectively. Although the agreement of the experimental data with Ref. [18] is surprisingly good, in general these theories are not expected to be very reliable for large wave vectors because they use the adiabatic approach, which neglects completely the interaction of the spin waves with the Stoner excitations. The broadening of the (acoustic) spin-wave peaks and the apparent absence of standing spin waves in the experimental spectra cannot be described by these adiabatic theories. For the description of these effects, a theory is necessary which takes into account the itinerant character of the electron spin. Such a (semiempirical) calculation has been performed for bulk hcp Co and a good agreement with the experiment was found for the acoustic spin-wave mode $[19,20]$. Unfortunately, a similar calculation for fcc $\mathrm{Co}$ is not available up to now. Recently, Savrasov presented a calculation of the dynamic spin susceptibility of bulk $\mathrm{Fe}$ and $\mathrm{Ni}$ based on a time-dependent $a b$ initio linear response density functional theory [21]. However, currently these calculations are computationally too demanding to be extended to thin films. For Fe films calculations of the spin susceptibility based on empirical tight-binding band structures show quantitative and qualitative differences to corresponding calculations using the adiabatic approach $[22,23]$. Such a calculation of the dynamic spin susceptibility for a Co film would certainly elucidate the question of whether the itinerant effects in Co are really much weaker compared to $\mathrm{Fe}$ or whether the surprisingly good agreement of the measured SPEELS spin-wave energies with the NN Heisenberg result is just accidental.

The cross section for spin-wave excitation depends very strongly on the energy $E_{0}$ of the incident electrons. Only below $E_{0} \approx 10 \mathrm{eV}$ can the spin wave be observed as a peak in the $I_{\downarrow}$ spectrum. Currently, we do not have a good explanation for this behavior.

In conclusion, we have shown that the dispersion of spin waves at surfaces and in ultrathin films can be measured by spin-polarized electron energy loss spectroscopy up to the surface Brillouin zone boundary. The spin-wave intensity in the EEL spectrum depends strongly on the energy of the incident electrons. For an 8 ML thick fcc Co film on $\mathrm{Cu}(001)$ the measured spinwave dispersion curve of the acoustic mode exhibits the behavior expected for a surface spin wave.

P.S. A. K. thanks the Alexander von Humboldt Foundation for financial support during the initial part of this work. We thank D. L. Mills for enlightening discussions and for making results available to us prior to publication.

*To whom correspondence should be addressed. Electronic address: vollme@mpi-halle.mpg.de

[1] Topics in Applied Physics, edited by B. Hillebrands and K. Ounadjela (Spinger-Verlag, Berlin, 2002), Vol. 83.

[2] A. Schreyer et al., J. Appl. Phys. 87, 5443 (2000).

[3] H. Hopster, R. Raue, and R. Clauberg, Phys. Rev. Lett. 53, 695 (1984).

[4] J. Kirschner, D. Rebenstorff, and H. Ibach, Phys. Rev. Lett. 53, 698 (1984).

[5] D. Venus and J. Kirschner, Phys. Rev. B 37, 2199 (1988).

[6] G. Vignale and K. S. Singwi, Phys. Rev. B 32, 2824 (1985).

[7] M. P. Gokhale, A. Ormeci, and D. L. Mills, Phys. Rev. B 46, 8978 (1992).

[8] M. Plihal, D. L. Mills, and J. Kirschner, Phys. Rev. Lett. 82, 2579 (1999).

[9] M. R. Vernoy and H. Hopster, Phys. Rev. B (to be published).

[10] H. Ibach, D. Bruchmann, R. Vollmer, M. Etzkorn, P. S. Anil Kumar, and J. Kirschner, Rev. Sci. Instrum. 74, 4089 (2003).

[11] D. T. Pierce et al., Rev. Sci. Instrum. 51, 478 (1980).

[12] A. K. Schmid et al., Phys. Rev. B 48, 2855 (1993).

[13] K.-P. Kämper, D. L. Abraham, and H. Hopster, Phys. Rev. B 45, 14335 (1992).

[14] R. N. Sinclair and B. N. Brockhouse, Phys. Rev. 120, 1638 (1960).

[15] D. L. Mills, in Surface Excitations, edited by V.M. Aranovich and R. Loudon (Elsevier, New York, 1984), Chap. 3, p. 379.

[16] M. P. Gokhale and D. L. Mills, Phys. Rev. B 49, 3880 (1994).

[17] M. Pajda et al., Phys. Rev. B 64, 174402 (2001).

[18] S. V. Halilov, H. Eschrig, A. Y. Perlov, and P. M. Oppeneer, Phys. Rev. B 58, 293 (1998).

[19] K. N. Trohidou, J. A. Blackman, and J. F. Cooke, Phys. Rev. Lett. 67, 2561 (1991).

[20] T. G. Perring, A. D. Taylor, and G. L. Squires, Physica (Amsterdam) 213B-214B, 348 (1995).

[21] S. Y. Savrasov, Phys. Rev. Lett. 81, 2570 (1998).

[22] H. Tang, M. Plihal, and D. L. Mills, J. Magn. Magn. Mater. 187, 23 (1998).

[23] R. B. Muniz and D. L. Mills, Phys. Rev. B 66, 174417 (2002). 